\title{
Three-month outcome of ziv-aflibercept for exudative age-related macular degeneration
}

\author{
Ahmad M Mansour, ${ }^{1,2}$ Jay Chhablani, ${ }^{3}$ Rafic S Antonios, ${ }^{1}$ Rohit Yogi, ${ }^{3}$ \\ Muhammad H Younis, ${ }^{1,2}$ Rola Dakroub, ${ }^{1,2}$ Hasan Chahine ${ }^{1}$
}

${ }^{1}$ Department of Ophthalmology, American University of Beirut, Beirut, Lebanon

${ }^{2}$ Department of Ophthalmology, Rafik Hariri University Hospital, Beirut, Lebanon

${ }^{3}$ Smt Kanuri Santhamma Centre for Vitreoretinal Diseases, LV Prasad Eye Institute, Hyderabad Andhra Pradesh, India

Correspondence to Dr Jay Chhablani, Smt. Kanuri Santhamma Centre for Vitreoretinal Diseases, LV Prasad Eye Institute, Hyderabad 500034, Andhra Pradesh, India; jay.chhablani@gmail.com

Received 29 December 2015 Revised 15 February 2016 Accepted 5 March 2016 Published Online First 30 March 2016

\begin{abstract}
Purpose In vitro and in vivo studies did not detect toxicity to the retinal pigment epithelium cells using intravitreal ziv-aflibercept. Our purpose is to ascertain the 3-month safety and efficacy in wet age-related macular degeneration (AMD) treated with intravitreal ziv-aflibercept.
\end{abstract}

Methods Prospectively, consecutive patients with wet AMD underwent ziv-aflibercept intravitreal injection (1.25 mg/0.05 mL) from March 2015 to November 2015. Monitoring of best-corrected visual acuity, intraocular inflammation, cataract progression and by spectral domain optical coherence tomography were carried out at baseline day 1, 1 week, 1 month, 2 months and 3 months after injections.

Results 30 eyes were treated (22 Caucasians, 8 Indians; 16 men, 14 women; 14 right eyes and 16 left eyes) with mean age of 74.3 years with 11 treatmentnaïve cases and 19 having had treatment-non-naïve. Best-corrected visual acuity improved from baseline $\log$ MAR $1.08-0.74$ at 1 week, 0.72 at 1 month, 0.67 at 2 months and 0.71 at 3 months $(p<0.001$ for all time periods). Central macular thickness in microns decreased from 332.8 to 302.0 at 1 week, 244.8 at 1 month, 229.0 at 2 months and 208.2 at 3 months ( $p<0.001$ for all time periods). There were no signs of intraocular inflammation, or change in lens status or increase in intraocular pressure throughout the study.

Conclusions Off label use of ziv-aflibercept improves visual acuity, without detectable ocular toxicity and offers a cheaper alternative to the same molecule aflibercept, especially in low/middle-income countries and in countries where aflibercept (Eylea) is not available.

Trial registration number NCT02486484.

\section{INTRODUCTION}

VIEW 1 and 2 (VEGF Trap-Eye: Investigation of Efficacy and Safety in Wet AMD) trials along with other studies ${ }^{12}$ have outlined aflibercept as an efficient anti-vascular endothelial growth factor (VEGF) in neovascular age-related macular degeneration (AMD) but its use in low/middle-income countries has been limited due to its high cost and its unavailability in some countries. In oncology, ${ }^{3}$ aflibercept has been approved by US Food and Drug Association (FDA) and is known as ziv-aflibercept or Zaltrap. We compounded ziv-aflibercept for use in patients who cannot afford Eylea, (FDA approved intravitreal aflibercept), on a compassionate basis on a long-term basis after lack of toxicity in cell cultures, ${ }^{4}$ animal studies, ${ }^{5}$ case reports ${ }^{6-8}$ and two phase I clinical studies. 910

The current clinical study reports the safety and efficacy of Zaltrap at 3 months after monthly intravitreal ziv-aflibercept in eyes with wet AMD.

\section{METHODS}

This is a prospective non-randomised two-centre study. This study was approved by the institutional review committee at both centres and adhered to the tenets of the declaration of Helsinki. Study duration at both centres was March 2015 to December 2015. Clinical trial registration number is NCT02486484. After detailed explanation of the protocol, all patients signed a formal consent before the initiation of therapy.

Inclusion criteria included naïve or previously treated neovascular AMD with the last injection of VEGF antagonist 4 months or more before enrolment. Exclusion criteria included disciform scar, polypoidal choroidal vasculopathy, diabetic retinopathy, infectious conjunctivitis, prior vitreous surgery and inability to commit for long-term follow-up. Best-corrected visual acuity (BCVA) was assessed by Early Treatment Diabetic Retinopathy Study (ETDRS) R chart (Precision Vision, La Salle, Illinois, USA) in Lebanon and Snellen charts in India. Central macular thickness (CMT), mean thickness in the central $1000 \mu \mathrm{m}$ diameter area) was done using optical coherence tomography (OCT) three-dimensional OCT-2000 FA plus Topcon (Topcon, Tokyo, Japan) in Lebanon, Cirrus (Carl Zeiss Meditec, Dublin, California, USA) in India. Intravenous fluorescein angiography was done at baseline using standard protocol. The same operator (AMM) performed the vision exam, OCT test and intravitreal injection in the Lebanese series while the certified Ophthalmology associates participated in the care of the patients in the Indian series. Intraocular pressures were measured using applanation tonometry.

Comprehensive ocular examination was performed at baseline, day 1 , week 1 , month 1 , month 2 and month 3. All patients underwent intravenous fluorescein angiography at baseline, however, SD-OCT was repeated at every visit. Ziv-aflibercept was prepared and injected according to standard protocols: compounding under sterile conditions with storage at $4^{\circ} \mathrm{C}$ for 4 weeks. ${ }^{8}$ Intravitreal injections were given every 4 weeks as $0.05 \mathrm{~mL}$ ziv-aflibercept (1.25 mg aflibercept) for 3 months. In eyes with fresh subfoveal bleed, additional injections at 2 weeks during the first month of therapy. Monitoring of intraocular inflammation and cataract progression was carried out at day 1 and 
Table 1 Clinical profile of the patients with wet AMD treated with intravitreal ziv-aflibercept

\begin{tabular}{ll}
\hline Variables & Results \\
\hline Mean age \pm SD (years) & $74.3 \pm 11.5$ \\
Gender (male:female) & $16: 14$ \\
Race (Caucasian:Indian) & $22: 8$ \\
Laterality (right:left eye) & $14: 16$ \\
Prior anti-VEGF injections (yes:no) & $19: 11$ \\
Mean number of prior anti-VEGF injections (range) & $5.2(1-15)$ \\
Phakic:pseudophakic & $15: 15$ \\
Smoker & $13(43 \%)$ \\
Coronary artery disease & $3(10 \%)$ \\
Diabetes mellitus & $8(27 \%)$ \\
Systemic hypertension & $6(20 \%)$ \\
Stroke & $1(3 \%)$ \\
\hline AMD, age-related macular degeneration; VEGF, vascular endothelial growth factor.
\end{tabular}

1 week, 1 month, 2 months and 3 months after injections using the slit lamp.

Statistical analyses were performed using SPSS V.22 (IBM, Chicago, Illinois, USA) and Wilcoxon signed-rank test with significance set at $\mathrm{p}$ value of 0.05 .

\section{RESULTS}

Thirty eyes with active choroidal neovascularisation secondary to AMD were treated (22 Caucasians, 8 Indians; 16 men, 14 women; 14 right eyes and 16 left eyes) with a mean age of 74.3 years. The cohort included 11 treatment-naïve eyes and 19 treatment-non-naïve eyes (table 1). Diabetes mellitus was present in eight patients, however, none of the eyes had diabetic retinopathy. BCVA improved from baseline logMAR 1.08 (approximate Snellen's equivalent 20/250) to 0.74 (Snellen's equivalent 20/100) at 1 week, 0.72 (Snellen's equivalent 20/ 100 ) at 1 month, 0.67 (Snellen's equivalent 20/80) at 2 months and 0.71 (Snellen's equivalent 20/100) at 3 months $(\mathrm{p}<0.001$ for all time periods) (table 2). CMT in microns decreased from 332.8 to 302.0 at 1 week, 244.8 at 1 month, 229.0 at 2 months and 208.2 at 3 months ( $p<0.001$ for all time periods). There were no signs of intraocular inflammation or change in lens status throughout the study. Intraocular pressure was unchanged at baseline and at 3 months $(12.8 \pm 2.3 \mathrm{~mm} \mathrm{Hg}$ vs 12.8 $\pm 2.2 \mathrm{~mm} \mathrm{Hg}$ ). Retinal pigment epithelial detachment and subretinal macular haemorrhage (figures 1 and 2) responded well to ziv-aflibercept given every 4 weeks.

Three eyes with fresh subfoveal haemorrhage received additional injections at 2 weeks during the first month of therapy. The treatment response in these three cases was as follows: first case improved from 20/400 (CMT $291 \mu$ ) to 20/20 (CMT $164 \mu$ ); second case from 20/600 (CMT $364 \mu$ ) to 20/40 (CMT $291 \mu$ ) and third case from 20/2000 (CMT $344 \mu$ ) to $20 / 100$ $(248 \mu)$ at the 3 -month follow-up.

No systemic side effects were noted during the study period.

\section{DISCUSSION}

Aflibercept is identical in structure to ziv-aflibercept; however, it contains different buffer solutions that are less irritating when injected intravitreally. Aflibercept comes as an iso-osmotic solution $(300 \mathrm{mOsm} / \mathrm{kg})$, while ziv-aflibercept has an osmolarity of $1000 \mathrm{mOsm} / \mathrm{kg}$ from the addition of sucrose. The issue of hyperosmolarity of ziv-aflibercept causing retinal toxicity was refuted in our previous publication. ${ }^{10}$ Injecting $0.05 \mathrm{~mL}$ of a hyperosmotic solution like ziv-aflibercept $(1000 \mathrm{mOsm} / \mathrm{kg})$ will raise the vitreous cavity osmolarity to $312 \mathrm{mOsm} / \mathrm{kg}$ from a baseline of $300 \mathrm{mOsm} / \mathrm{kg}$ or $4 \%$ increase that is within the physiological range.

Other issues raised when off label ziv-aflibercept are used in a compounded manner include safety, potential ocular toxicity (cataract, glaucoma and retinal damage), clinical efficacy (short and long-term) assessed by visual gain and CMT, stability profile of compounded drug and finally the cost. There was no evidence of ocular toxicity in the current study throughout the 90-day observation period. Malik et al evaluated apoptosis in human retinal pigment epithelium cells (ARPE-19) exposed for $24 \mathrm{~h}$ to clinically relevant doses of four anti-VEGF drugs and found no mitochondrial toxicity with either ranibizumab or aflibercept and mild mitochondrial toxicity with bevacizumab and ziv-aflibercept. De Oliveira Dias et al injected ziv-aflibercept in a refractory case of exudative AMD with visual improvement at 1 month along with decrease in both intraretinal and subretinal

Table 2 CMT and BCVA before and at 1 week, 1 month, 2 months, 3 months after injection of intravitreal ziv-aflibercept ( $p$ value is measured against baseline values using the Wilcoxon signed-rank test)

\begin{tabular}{|c|c|c|c|c|c|}
\hline & Baseline & 1 Week* & 1 Month & 2 Months & 3 Months \\
\hline \multicolumn{6}{|c|}{ Combined naïve and non-naïve cases } \\
\hline Number of eyes & 30 & 22 & 29 & 26 & 22 \\
\hline $\mathrm{CMT}$ mean $\pm \mathrm{SD}$ & $332.8 \pm 135.1$ & $\begin{array}{l}302.0 \pm 112.4 \\
(p<0.001)\end{array}$ & $\begin{array}{l}244.8 \pm 77.3 \\
(p<0.001)\end{array}$ & $\begin{array}{l}229.0 \pm 60.5 \\
(p<0.001)\end{array}$ & $\begin{array}{l}208.2 \pm 50.91 \\
(p<0.001)\end{array}$ \\
\hline $\mathrm{BCVA} \log \mathrm{MAR} \pm \mathrm{SD}$ (range) & $\begin{array}{l}1.08 \pm 0.67 \\
(0.10-2.50)\end{array}$ & $\begin{array}{l}0.74 \pm 0.52 \\
(0.04-2.00) \\
(p=0.009)\end{array}$ & $\begin{array}{l}0.72 \pm 0.47 \\
(0.00-1.60) \\
(p<0.001)\end{array}$ & $\begin{array}{l}0.66 \pm 0.50 \\
(0.00-1.60) \\
(p=0.001)\end{array}$ & $\begin{array}{l}0.71 \pm 0.54 \\
(0.00-2.00) \\
(p=0.002)\end{array}$ \\
\hline \multicolumn{6}{|l|}{ Treatment-naïve eyes } \\
\hline Number of eyes & 11 & 10 & 11 & 10 & 9 \\
\hline $\mathrm{CMT}$ mean $\pm \mathrm{SD}$ & $415.5 \pm 144.6$ & $\begin{array}{l}345.2 \pm 114.3 \\
(p=0.138)\end{array}$ & $\begin{array}{l}301.5 \pm 86.2 \\
(p=0.021)\end{array}$ & $\begin{array}{l}254.3 \pm 73.4 \\
(p=0.013)\end{array}$ & $\begin{array}{l}212.7 \pm 51.0 \\
(p=0.008)\end{array}$ \\
\hline $\mathrm{BCVA} \log \mathrm{MAR} \pm \mathrm{SD}$ (range) & $\begin{array}{l}1.38 \pm 0.57 \\
(0.54-2.50)\end{array}$ & $\begin{array}{l}0.76 \pm 0.59 \\
(0.04-2.00) \\
(p=0.005)\end{array}$ & $\begin{array}{l}0.82 \pm 0.50 \\
(0.30-1.60) \\
(p=0.005)\end{array}$ & $\begin{array}{l}0.78 \pm 0.58 \\
(0.18-1.60) \\
(p=0.005)\end{array}$ & $\begin{array}{l}0.68 \pm 0.46 \\
(0.00-1.60) \\
(p=0.008)\end{array}$ \\
\hline
\end{tabular}

*Eight subjects missed the 1-week follow-up. On the other side, a different set of eight subjects did not complete the 3-month follow-up.

BCVA, best-corrected visual acuity; CMT, central macular thickness. 


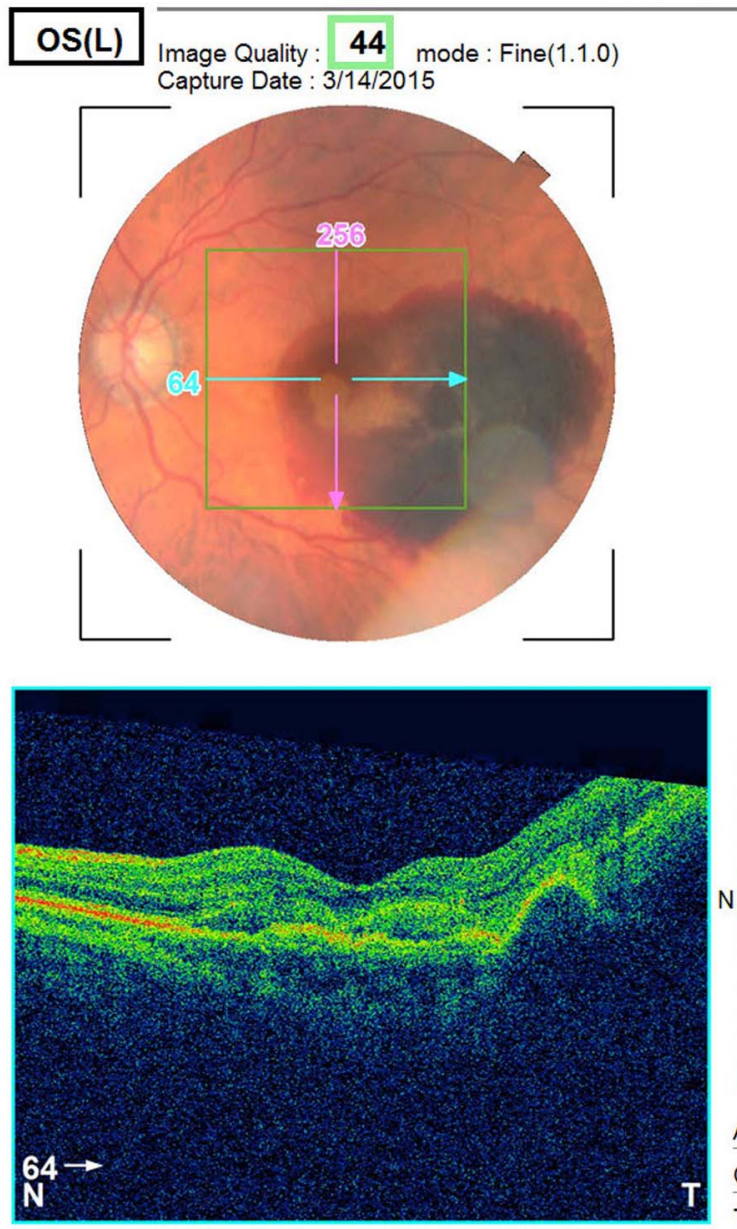

Retinal thickness map ILM - RPE / Red-free

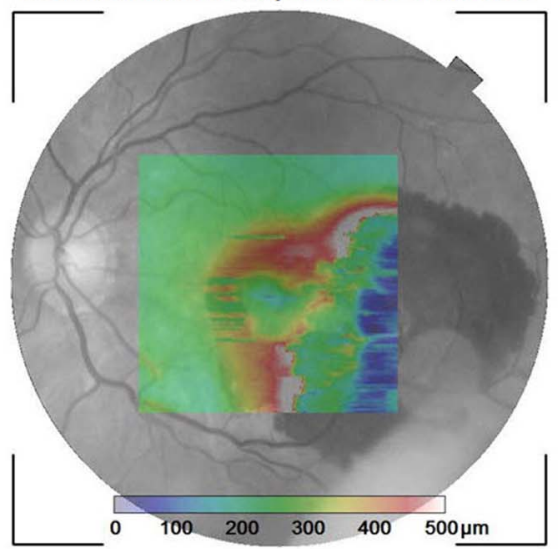

Retinal thickness ILM - RPE $(\mu \mathrm{m})$
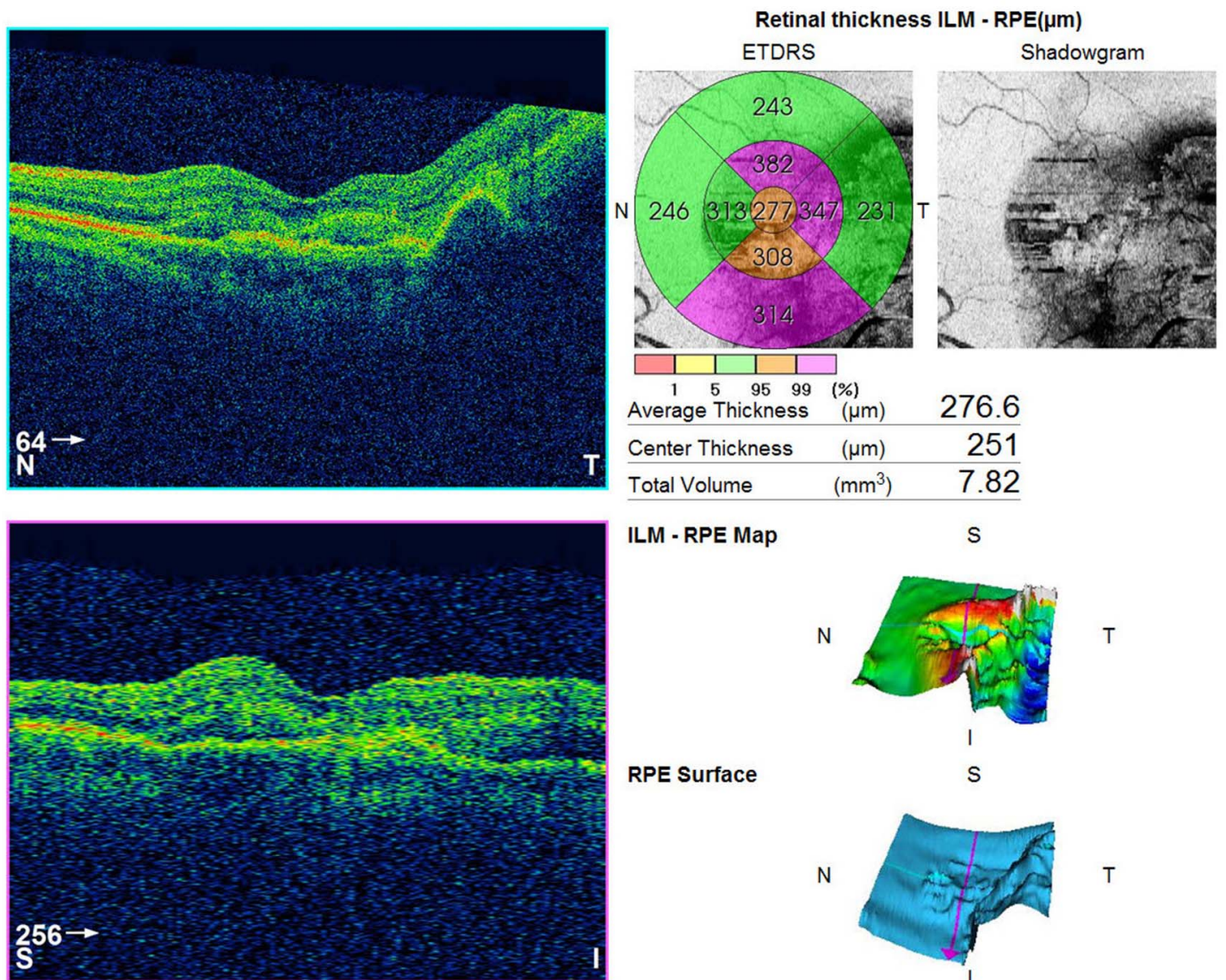

ILM - RPE Map

$\mathrm{S}$

RPE Surface
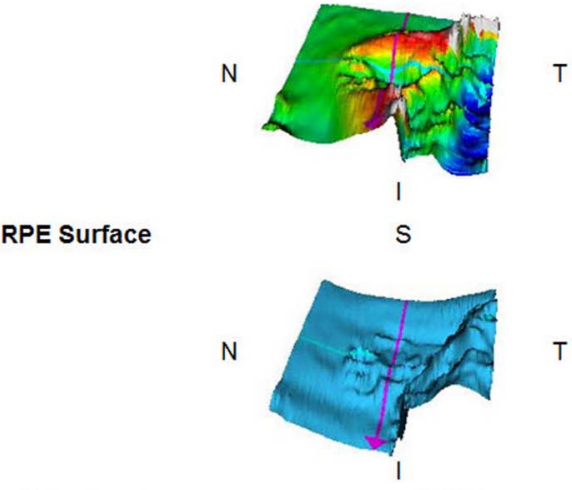

Signature:

Date :

Figure 1 Left panel shows the left eye images of an 84-year-old Lebanese man with sudden loss of vision of a few days duration with best-corrected visual acuity of 20/400. Patient was a smoker with coronary artery disease and atrial fibrillation on three oral anticoagulants after coronary bypass surgery. Indocyanine green angiography rules out the presence of polypoidal choroidal vasculopathy. Fundus photograph shows fresh subretinal blood secondary to wet age-related macular degeneration. Patient underwent three monthly intravitreal injections of ziv-aflibercept $(1.25 \mathrm{mg} / 0.05 \mathrm{~mL})$ with additional injection at 2 weeks after the first injection. ETDRS, Early Treatment Diabetic Retinopathy Study. ILM, internal limiting membrane; RPE, retinal pigment epithelium.

fluid. Microperimetric improvement also occurred while electroretinogram recording was unchanged. Similarly, Chhablani ${ }^{6}$ used ziv-aflibercept in a patient with recalcitrant bilateral central retinal vein occlusion. After 1 month, CMT dramatically decreased from $834 \mu$ in the right eye and $938 \mu$ in the left eye to $193 \mu$ (right eye) and $232 \mu$ (left eye). Videkar et al ${ }^{8}$ reported a case of polypoidal choroidal vasculopathy resistant to photodynamic therapy and ranibizumab, which responded well to a single intravitreal ziv-aflibercept with resolution of subretinal by OCT performed 1 month postinjection. Ziv-aflibercept demonstrated short-term safety and efficacy after intravitreal administration for a small series of neovascular AMD. ${ }^{10}$ In four such patients, the height of the foveolar detachment of the retinal pigment epithelium decreased from a mean of $583 \mu$ to a 

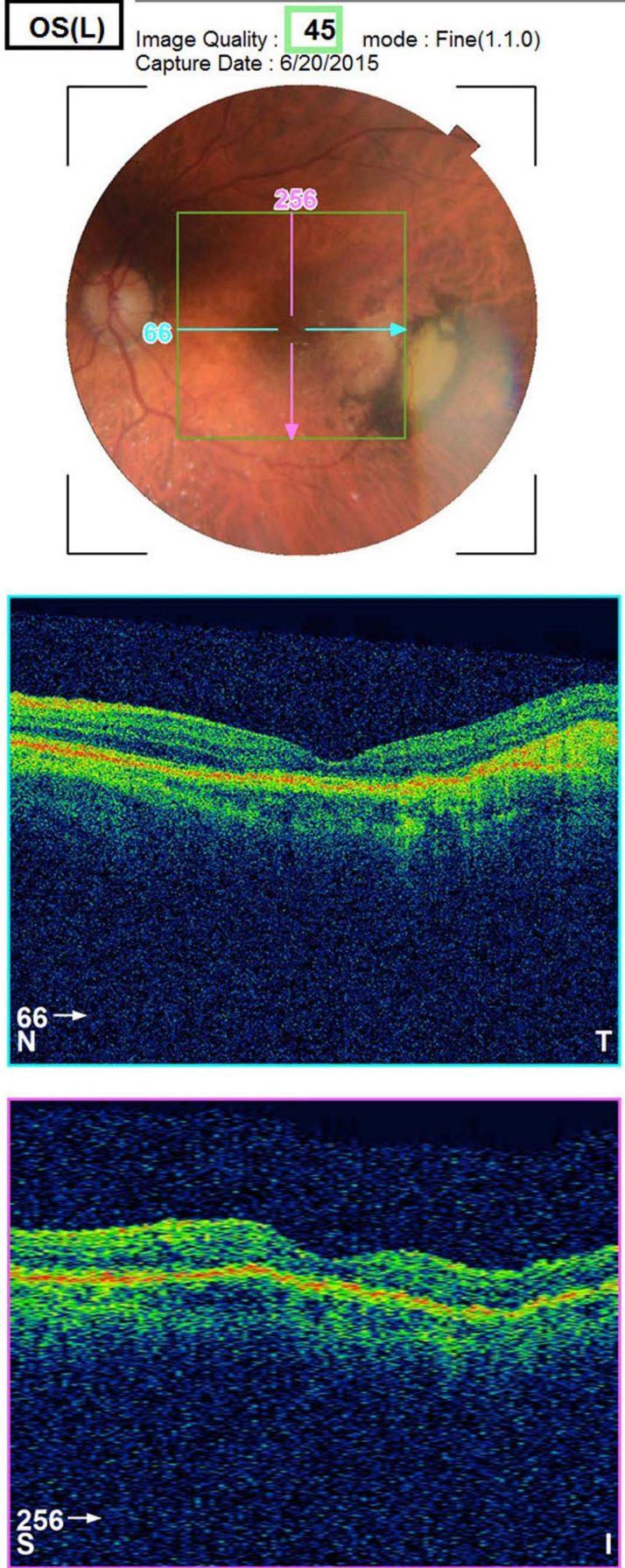

Comments :
Retinal thickness map ILM - RPE / Red-free

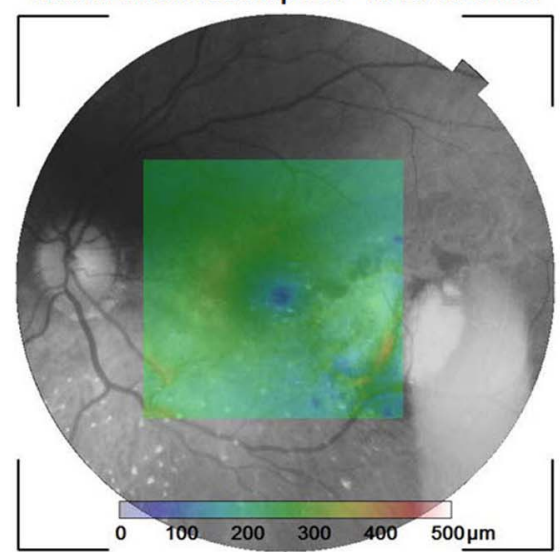

Retinal thickness ILM - RPE( $\mu \mathrm{m})$ ETDRS
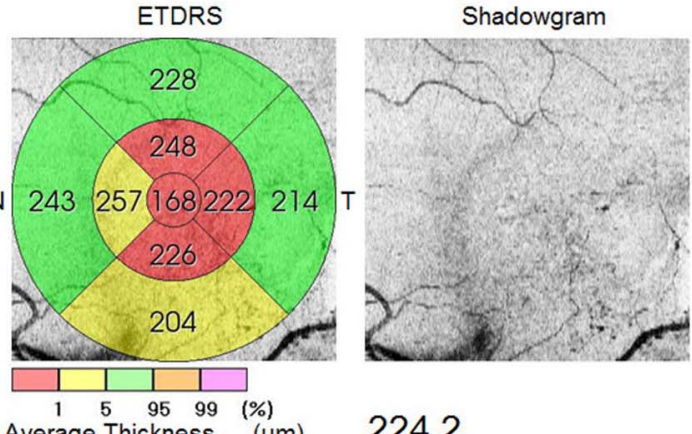

224.2

\begin{tabular}{lcr}
\hline Center Thickness & $(\mu \mathrm{m})$ & 153 \\
\hline Total Volume & $\left(\mathrm{mm}^{3}\right)$ & 6.34 \\
\hline
\end{tabular}

ILM - RPE Map

S

N

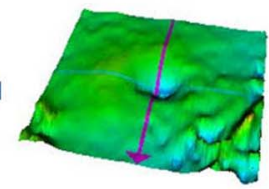

I

RPE Surface

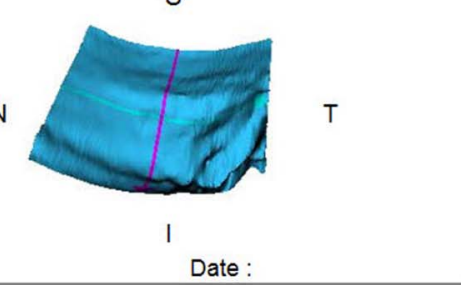

Figure 2 At 3-month follow-up of the same patient, described in figure 1, his visual acuity improved to 20/20 with disappearance of subretinal haemorrhage and recovery of foveal contour. ETDRS, Early Treatment Diabetic Retinopathy Study. ILM, internal limiting membrane; RPE, retinal pigment epithelium.

final mean of $398 \mu 1$ week after injection. ${ }^{10}$ More recently, Chhablani et $a l^{9}$ detected no change in electroretinogram recording 1 month after a single intravitreal ziv-aflibercept in 12 eyes with wet AMD.

In VIEW 1 and 2 (VEGF Trap-Eye: Investigation of Efficacy and Safety in Wet AMD) trials, patients were randomised to 1 of 4 groups: $^{1}{ }^{2}$ aflibercept $2 \mathrm{mg}$ every 8 weeks (after three initial monthly doses), aflibercept $2 \mathrm{mg}$ every 4 weeks, aflibercept $0.5 \mathrm{mg}$ every 4 weeks and ranibizumab $0.5 \mathrm{mg}$ every 4 weeks.
The four groups had comparable primary endpoint, that is, the proportion of patients maintaining vision (defined as losing $<15$ letters on an ETDRS chart) at 52 weeks. In the current study, the dose used was $1.25 \mathrm{mg}$ of ziv-aflibercept (halfway between the $2 \mathrm{mg}$ and $0.5 \mathrm{mg}$ doses studied for aflibercept). Hence, the current dose for ziv-aflibercept falls within the proven therapeutic doses of aflibercept as shown by the 3-month data. Additional support for the dosage issue comes from a case report by Nagiel et $a l^{11}$ depicting resolution of a giant pigment 
epithelial detachment using half-dose of aflibercept $(1 \mathrm{mg})$. In our treatment-naïve and fresh wet AMD series, mean CMT decreased significantly by $202.8 \mu$ and vision improved by 0.60 logMAR (approximate six lines improvement on ETDRS chart) at the 3-month follow-up from $20 / 480$ to $20 / 96$. Treatment-non-naïve eyes in our series had much more modest visual gain of $0.5 \log$ MAR (approximate five lines improvement on ETDRS chart) and foveal flattening of $87.2 \mu$, reflecting poorer anti-VEGF response in the chronic phase of the disease. $^{12} 13$ To achieve a dose of $2 \mathrm{mg}$ of aflibercept would require injecting $0.08 \mathrm{~mL}$ of ziv-aflibercept that could lead to prolonged increased intraocular pressure. With the dose of $2 \mathrm{mg}$, that is, $0.08 \mathrm{~mL}$ of ziv-aflibercept, high intraocular pressure due to higher volume may be a concern.

The treatment-naïve series showed large gains in vision $(0.6$ logMAR) after injections. VIEW studies ${ }^{1} 2$ enrolled eyes with vision better or equal to 6/120 (20/400) while we included eyes with poor baseline acuity. The large visual gain in our series relates to the enrolment of eyes with initial poor vision and possibly also to the prompt ${ }^{12-16}$ and intense initial treatment in these cases (three eyes with fresh subfoveal haemorrhage received biweekly injection in the first month). Jain et $a l^{15}$ treated 14 eyes with thick subfoveal haemorrhage within 1 week of visual loss (13 eyes with bevacizumab and one eye with ranibizumab) and found $0.54 \log$ MAR visual improvement at 6 months follow-up. Kim et $a l^{14}$ found $0.42 \operatorname{logMAR}$ visual improvement in 91 eyes with submacular haemorrhage from AMD and correlated visual improvement with longer duration of symptoms.

The compounded ziv-aflibercept drug was stable for 4 weeks as shown indirectly by good clinical response in the current study and directly by VEGF assays showing no loss of VEGF blockade at 1-month storage. ${ }^{10}$ The current study was carried out using polycarbonate barrel syringes that have poor binding to protein and excellent safety profile. ${ }^{10} 16$

Currently, bevacizumab is the most cost-effective medication for control of wet AMD compared with ranibizumab (40 times cheaper) or aflibercept. ${ }^{17-19}$ The actual compounded cost is 60 times less for ziv-aflibercept than for aflibercept, if the $4 \mathrm{~mL}$ ziv-aflibercept phial is divided into 40 aliquots $(4 \mathrm{~mL}$ Zaltrap $25 \mathrm{mg} / \mathrm{mL}$ phial costs $\$ 512$ while Eylea costs \$1200). In view of the excellent stability profile of the $\operatorname{drug}^{10}$ and short-term clinical safety, ${ }^{9}$ ziv-aflibercept would appear, based on the 3-month data, to be even more cost-effective than bevacizumab $(100 \mathrm{mg}$, $4 \mathrm{~mL}$ phial costs $\$ 660)$. $^{10}$

The limitations of the current study include the small number of eyes treated; small sample bias overall; irregular short duration of follow-up; and one-armed open label design. We did not perform any electroretinographic tests to confirm any toxicity at 3 months. However, our study provides satisfactory data on safety and efficacy of Zaltrap in eyes with wet AMD after three monthly injections.

In conclusion, off label use of ziv-aflibercept over 3-month period improves visual acuity, without detectable ocular toxicity and offers a cheaper alternative to the same molecule aflibercept, especially in low/middle-income countries and in countries where aflibercept (Eylea) is not available. Further studies on long-term safety with multiple injections in various retinal diseases are underway to explore ziv-aflibercept as an alternative intravitreal anti-VEGF injection.

Contributors Substantial contributions to the conception or design of the work, or the acquisition, analysis or interpretation of data (AMM, JC, RSA, RY, MHY, RD and $\mathrm{HC}$ ). Drafting the work or revising it critically for important intellectual content (AMM, JC, RSA, RD and HC). Final approval of the version published (AMM, JC, RSA, RY, MHY, RD and HC). Agreement to be accountable for all aspects of the work in ensuring that questions related to the accuracy or integrity of any part of the work are appropriately investigated and resolved (AMM and JC).

Competing interests None declared.

Patient consent Obtained.

Ethics approval Local institutional ethics committee.

Provenance and peer review Not commissioned; externally peer reviewed.

\section{REFERENCES}

1 Schmid MK, Bachmann LM, Fas L, et al. Efficacy and adverse events of aflibercept, ranibizumab and bevacizumab in age-related macular degeneration: a trade-off analysis. Br J Ophthalmol 2015;99:141-6.

2 Schmidt-Erfurth U, Kaiser PK, Korobelnik JF, et al. Intravitreal aflibercept injection for neovascular age-related macular degeneration: ninety-six-week results of the VIEW studies. Ophthalmology 2014;121:193-201.

3 Chung C, Pherwani N. Ziv-aflibercept: a novel angiogenesis inhibitor for the treatment of metastatic colorectal cancer. Am J Health Syst Pharm 2013;70:1887-96.

4 Malik D, Tarek M, Caceres del Carpio J, et al. Safety profiles of anti-VEGF drugs: bevacizumab, ranibizumab, aflibercept and ziv-aflibercept on human retinal pigment epithelium cells in culture. Br J Ophthalmol 2014;98 Suppl 1(Suppl 1):i11-16.

5 de Oliveira Dias JR, Badaró E, Novais EA, et al. Preclinical investigations of intravitreal ziv-aflibercept. Ophthalmic Surg Lasers Imaging Retina 2014;45:577-84.

6 Chhablani J. Intravitreal ziv-aflibercept for recurrent macular edema secondary to central retinal venous occlusion. Indian J Ophthalmol 2015;63:469-70.

7 de Oliveira Dias JR, Xavier CO, Maia A, et al. Intravitreal injection of ziv-aflibercept in patient with refractory age-related macular degeneration. Ophthalmic Surg Lasers Imaging Retina 2015;46:91-4.

8 Videkar C, Kapoor A, Chhablani J, et al. Ziv-aflibercept: a novel option for the treatment of polypoidal choroidal vasculopathy. BMJ Case Rep 2015;2015.

9 Chhablani J, Narayanan R, Mathai A, et al. Short-term safety profile of intravitreal ziv-aflibercept. Retina Published Online First: 11 Dec 2015; doi:10.1097/ IAE.0000000000000913

10 Mansour AM, Al-Ghadban SI, Yunis MH, et al. Ziv-aflibercept in macular disease. Br J Ophthalmol 2015;99:1055-9.

11 Nagiel A, Sadda SR, Schwartz SD, et al. Resolution of a giant pigment epithelial detachment with half-dose aflibercept. Retin Cases Brief Rep 2015;9:269-72.

12 Ziemssen F, Bertelmann T, Hufenbach $U$, et al. [Delayed treatment initiation of more than 2 weeks: relevance for possible gain of visual acuity after anti-VEGF therapy under real life conditions (interim analysis of the prospective OCEAN study)]. Ophthalmologe 2016;113:143-51.

$13 \mathrm{Lim} \mathrm{JH}$, Wickremasinghe SS, Xie J, et al. Delay to treatment and visual outcomes in patients treated with anti-vascular endothelial growth factor for age-related macular degeneration. Am J Ophthalmol 2012;153:678-86, 86 e1-2.

$14 \mathrm{Kim} \mathrm{JH}$, Chang YS, Kim JW, et al. Intravitreal anti-vascular endothelial growth factor for submacular hemorrhage from choroidal neovascularization. Ophthalmology 2014; 121:926-35.

15 Jain S, Kishore K, Sharma YR. Intravitreal anti-VEGF monotherapy for thick submacular hemorrhage of less than 1 week duration secondary to neovascular age-related macular degeneration. Indian J Ophthalmol 2013;61:490-6.

16 Bailey C, Aloumanis V, Walker $B$, et al. Stability of preoperative cataract surgery gel in polycarbonate syringes. Int J Pharm Compd 2009;13:564-8.

17 Stein JD, Newman-Casey PA, Mrinalini T, et al. Cost-effectiveness of bevacizumab and ranibizumab for newly diagnosed neovascular macular degeneration. Ophthalmology 2014;121:936-45.

18 Silver J. Drugs for macular degeneration, price discrimination, and Medicare's responsibility not to overpay. Jama 2014;312:23-4.

19 Raftery J, Clegg A, Jones J, et al. Ranibizumab (Lucentis) versus bevacizumab (Avastin): modelling cost effectiveness. Br J Ophthalmol 2007;91:1244-6. 